Cahiers de recherches médiévales

\title{
Un baron révolté est-il un hors-la-loi ?
}

Droit et violence dans Girart de Roussillon

\section{Philippe Haugeard}

\section{(2) OpenEdition \\ Journals}

Édition électronique

URL : https://journals.openedition.org/crm/11712

DOI : $10.4000 / \mathrm{crm} .11712$

ISSN : 1955-2424

Éditeur

Honoré Champion

Édition imprimée

Date de publication : 20 novembre 2009

Pagination : 279-291

ISSN : 1272-9752

\section{Référence électronique}

Philippe Haugeard, "Un baron révolté est-il un hors-la-loi ? », Cahiers de recherches médiévales [En ligne], 18 | 2009, mis en ligne le 15 décembre 2012, consulté le 15 décembre 2022. URL : http:// journals.openedition.org/crm/11712; DOI : https://doi.org/10.4000/crm.11712 


\title{
酶M
}

\section{Un baron révolté est-il un hors la loi? Droit et violence dans Girart de Roussillon}

\begin{abstract}
In the old French epics of revolt, is the rebel vassal an outlaw? This question, which has a heuristic function, sets up the problem of the relations between law, right and violence. The study focuses on Girart of Roussillon, an epic of the twelfth century, in which the numerous scenes of discussion and debate show that the word droit (right) does not refer in fact to our modern and juridical notion. Girart of Roussillon represents a world of warriors and lords in which right is not yet law and in which, for that reason, violence born of the will to power cannot be contained.
\end{abstract}

Résumé : Un baron révolté est-il un hors-la-loi? La question, à laquelle nous attribuons une fonction heuristique, vise à mettre en relief une caractéristique essentielle de la société aristocratique représentée dans les plus anciennes chansons de révolte, à savoir l'illusion trompeuse de l'existence d'un droit régissant les comportements et définissant les droits et les devoirs de chacun en fonction de sa place à l'intérieur de la structure féodale. L'étude porte sur Girart de Roussillon, une chanson de geste du XII ${ }^{e}$ siècle, dans laquelle les nombreuses scènes de discussion et de délibération font apparaître que la forte présence du mot droit n'implique pas nécessairement l'existence d'un droit, au sens moderne et juridique du terme. C'est parce que le droit n'est pas encore la loi que la violence suscitée par la volonté de puissance des protagonistes ne peut être contenue.

Un baron révolté est-il un hors-la-loi ? La question peut paraître de faible rendement de prime abord. Apposer le concept de hors-la-loi au type épique du vassal rebelle ne semble pas en effet autre chose que soulever une nouvelle fois un problème qui a déjà été amplement traité par la critique, celui de la responsabilité du héros dans le déchaînement de violences que provoque sa révolte dans les chansons de geste dites «des vassaux rebelles ». Les termes de ce problème sont désormais solidement établis : victime d'un déni de justice ou d'un abus de pouvoir, le héros entre en conflit avec la puissance royale pour défendre sa personne et ses droits; le conflit s'éternise et s'intensifie en violence, les deux ennemis étant animés par la même démesure et le même orgueil; si la responsabilité du conflit est d'abord, indiscutablement, du côté du souverain, la question juridique des droits et des torts se complique par la suite, le baron révolté commettant à son tour, lui ou ses alliés, des crimes qui le condamnent et qui ne font qu'attiser la haine du souverain ; en dépit de la négativité du souverain en tant que personne, les chansons de révolte ne remettent pas en cause la royauté comme institution, à laquelle elles sont favorables et qu'elles font triompher finalement de la rébellion du vassal ${ }^{1}$. Une idéologie royale sous-tend ainsi la rédaction de textes qui inscrivent la révolte et la violence de ses

\footnotetext{
${ }^{1} \mathrm{M}$. de Combarieu, L'idéal humain et l'expérience morale chez les héros des chansons de geste, Université de Provence, 1979, t. 1, p. 134-177 et p. 203-216 ; D. Boutet, Charlemagne et Arthur, ou le roi imaginaire, Paris, Champion, 1992, p. 368-424.
}

Cahiers de Recherches Médiévales, 18, 2009 
développements dans le cadre juridique de la féodalité, non pas pour trancher les questions de droit, mais pour tirer parti de la complexité des situations et accroître la tension dramatique d'un conflit dont le ressort premier n'est en réalité ni politique, ni juridique, mais proprement psychologique :

Quelles que soient les modalités narratives de l'ouverture des crises [...], c'est toujours l'orgueil, le sens du lignage ou une insuffisante maitrise de soi qui sont les vrais principes générateurs des conflits. Le roi, le vassal, éventuellement les traîtres, le droit, la justice sont les éléments cristallisateurs et les supports de la crise dans cet univers du politique où se meut normalement la chanson de geste. Ils jouent, bien entendu, un rôle important : mais ils ne constituent peut-être pas le vrai sujet de ces œuvres. La surdétermination des conflits, dans tous les cas de figure que nous avons pu examiner, le laisse déjà supposer : en compliquant la question des responsabilités, et en soulignant l'échec des voies judiciaires, les auteurs prouvent qu'ils ne cherchent pas à illustrer des situations emblématiques pour dire le droit ou le tort des parties $^{2}$.

Après de telles analyses on voit mal de prime abord ce que le concept de hors-la-loi pourrait apporter de plus à la réflexion critique sur la révolte du vassal réflexion critique qui ne pose d'ailleurs pas la question en termes de loi mais de droit.

Mais tout l'intérêt du concept de hors-la-loi se trouve justement là, dans l'obligation qu'il génère de prendre en compte une notion de toute évidence jugée inappropriée par la critique (c'est un constat, pas un reproche) pour interroger les comportements des protagonistes des chansons de révolte, individus qui se réfèrent volontiers au droit et à l'éthique pour justifier leurs actes et leurs choix mais qui n'apparaissent pas en effet soumis à un corps unique de règles et de principes à valeur universelle et constituant pour eux un ordre supérieur contraignant, reconnu par tous et s'imposant à tous de la même façon. La périphrase « hors-la-loi » suppose au contraire un monde régi par un ordre clair, repérable et immédiatement identifiable - cette loi qu'on peut respecter ou enfreindre, ensemble de prescriptions et d'interdictions devenu comme tangible, constituant un espace presque physique à l'intérieur ou à l'extérieur duquel l'individu choisit de vivre ou d'agir, et qui, s'imposant à tous sans abolir la liberté de chacun de s'y soumettre ou de s'en affranchir, dessine une ligne de partage clairement visible entre les justes, les honnêtes gens d'un côté, l'immense majorité des hommes, et les mauvais, les vils criminels de l'autre côté, une poignée d'individus qui font exception et qui sont l'objet d'une condamnation générale et unanime. Inadéquat donc, a priori, pour qualifier le héros des chansons de révolte (comme d'ailleurs tous leurs autres protagonistes), le concept de hors-la-loi remplira ici une fonction essentiellement heuristique : en effet, la question du baron rebelle hors-la-loi constitue pour nous une hypothèse de recherche à partir de laquelle nous croyons possible d'approfondir notre connaissance des dimensions juridiques, éthiques et psychologiques des conflits sanglants qui font la matière des chansons du cycle des barons révoltés,

${ }^{2}$ D. Boutet, ibid., p. 380. 
œuvres qui décrivent une société féodale profondément travaillée par la question du droit, mais où le droit n'est pas encore la loi.

L'étude portera sur Girart de Roussillon ${ }^{3}$. Pour deux raisons. La première est que l'auteur marque peu d'intérêt pour les récits de bataille, préférant à l'héroïsme épique de la guerre les développements à caractère juridique : les scènes de conseil, d'ambassade et de négociation sont nombreuses et occupent une place exceptionnelle par rapport à la production épique contemporaine ; le texte propose ainsi une ample matière à partir de laquelle il est possible de mieux cerner la réalité de ce qu'on appelle communément le droit féodal, notion à laquelle la critique littéraire recourt volontiers, sans toujours s'interroger suffisamment sur ce dont il s'agit exactement au XII ${ }^{\mathrm{e}}$ siècle. La deuxième raison est que le héros finit par porter seul la responsabilité du déchaînement de violence que provoque sa résistance à l'abus de pouvoir que représente la tentative de Charles Martel de reprendre par la force à Girart les terres qu'il lui avait concédées auparavant en alleux; le tort, dans le déclenchement de l'interminable et désastreux conflit qui oppose les deux protagonistes, est indiscutablement du côté du roi mais c'est Girart que l'on voit, à un certain moment du récit, devoir prendre la fuite, se cacher et faire l'expérience d'une souffrance physique et morale puis d'une déchéance sociale explicitement données comme le châtiment de son orgueil - cet orgueil de grand seigneur qui l'a conduit à contester l'autorité du souverain et, ce faisant, à mettre à feu et à sang le royaume, emporté parfois par une haine qui l'aveugle et le pousse à commettre des gestes impies. Banni par le roi, maudit des hommes, châtié par Dieu, le héros devient un paria et un réprouvé qui a profondément porté atteinte, par sa démesure, à l'ordre des choses. Parmi les différentes chansons des barons rebelles, Girart de Roussillon nous paraît donc particulièrement propice pour poser la question du rapport entre le droit, censé régir les relations humaines dans le sens de l'harmonie sociale, et la violence - violence dont la responsabilité incombe aux individus mais dont le déchaînement est favorisé par la nature même du droit auquel se réfèrent ces mêmes individus, un droit qui est avant tout une morale de classe et qui s'avère incapable de brider la volonté de puissance des principaux protagonistes du récit : le droit, omniprésent pourtant dans Girart de Roussillon, ne fait donc pas force de loi, et cela parce qu'il n'est pas encore de même nature que la loi - c'est ce que nous voudrions montrer4.

Les occurrences du mot «droit» sont nombreuses dans Girart de Roussillon; elles apparaissent principalement, comme on s'en doute, dans les diverses scènes de

\footnotetext{
${ }^{3}$ Il convient de rappeler ici l'existence de la belle et riche étude consacrée par Pierre Le Gentil à cette chanson, "Girart de Roussillon, sens et structure du poème ", Romania, 1957, p. 328389 et p. $463-510$.

${ }^{4}$ Le texte de Girart de Roussillon que nous avons utilisé et que nous citerons est celui établi par W. M. Hackett, 3 vol., Paris, SATF, 1953-1955, texte reproduit, traduit, présenté et annoté par Micheline de Combarieu du Grès et Gérard Gouiran pour La chanson de Girart de Roussillon, Paris, Librairie Générale Française, Coll. "Lettres Gothiques », 1993. Vu la redoutable difficulté du texte, nous donnerons parfois, en note ou dans le texte, la traduction des passages cités que proposent M. de Combarieu et G. Gouiran. Nous avons aussi consulté la traduction de Paul Meyer, Girart de Roussillon, Paris, Champion, 1884.
} 
conseil, d'ambassade et de négociation que propose le texte. Ces scènes sont généralement l'occasion de débats au cours desquels les participants cherchent à trouver ou à faire accepter des solutions de compromis prenant en compte les torts et les droits de chacun des deux protagonistes, torts et droits qui évoluent au gré des situations nouvelles inventées par le récit mais qui restent inscrits la plupart du temps dans un cadre fixe et immuable, celui de la féodalité. Les questions de «droit» soulevées dans ces scènes relèvent majoritairement de ce qu'on appelle communément le droit féodal; l'expression est pourtant lourde d'ambiguïté pour le $\mathrm{XII}^{\mathrm{e}}$ siècle. L'expression «droit féodal » laisserait en effet entendre l'existence de quelque chose comme une législation ou une réglementation, à valeur générale certes, mais définissant avec un minimum de précision les divers aspects de la relation qui lie seigneurs et vassaux; il n'en est rien à l'époque considérée, comme le rappelle Gérard Giordanengo: "Le droit féodal est jusqu'à la fin du XII siècle une affaire de conventions (les fameuses convenientice) et les difficultés sont réglées au coup par coup. Que les problèmes soient à peu près partout les mêmes, ils concernent en général les successions et les partages, les sanctions au manquement de fidélité du vassal ou les obligations militaires, et ne puissent donner lieu qu'à un nombre forcément limité de solutions ne doit pas laisser croire à des règles générales, connues et acceptées par tous, servant de référence : la force et l'arbitrage décident $[. ..] . \|^{5} \mathrm{Ce}$ qui caractérise les relations entre le seigneur et le vassal à l'époque de la composition de Girart de Roussillon, ce serait donc plus un grand vide juridique qu'un trop plein de prescriptions et d'interdictions : seigneur et vassal sont certes liés par des obligations réciproques, par exemple de ne pas se faire la guerre, mais le contenu positif du droit féodal se réduit à des devoirs - auxilium et consilium - qui ne sont généralement pas l'objet d'une définition stricte ou d'une codification précise, sauf dans le cadre de conventions particulières, lesquelles n'engagent jamais que deux contractants, sans avoir de valeur générale, et ne pouvant pas dès lors servir de référence universelle $\mathrm{e}^{6}$

Dans Girart de Roussillon, comme dans les conflits féodaux de l'époque, la guerre et la négociation suppléent l'absence de ces «règles générales, connues et acceptées par tous » qui constituent désormais le Droit; cette absence de droit, au sens moderne du terme, n'empêche cependant pas la fréquence du mot, lequel semble bien posséder, dans l'esprit des différents acteurs du récit, une véritable dimension juridique, en cela en tout cas qu'ils lui reconnaissent une existence autonome et une valeur contraignante. Pour cerner le sens des principaux emplois de la notion de droit dans le texte, le mieux serait de commencer sans doute par ce que disent les dictionnaires d'Ancien Français. Pour le mot «droit», dans son sens juridique, 1'Altfranzösisches Wörterbuch de Tobler-Lommatzsch est comme d'habitude précieux pour le nombre des exemples proposés et l'explicitation du sens des diverses locutions où il apparaît («avoir droit, faire droit, querre droit, jugier droit, etc. ») mais c'est au Dictionnaire de l'ancienne langue française de Godefroy

\footnotetext{
${ }^{5}$ G. Giordanengo, «Coutume et droit féodal en France (XII $-\mathrm{XIV}^{\mathrm{e}}$ siècle) », Féodalités et droits savants dans le Midi Médiéval, Aldershot (GB), 1992, chap. XV, p. 220-221.

${ }^{6} \mathrm{P}$. Bonnassie, "Les conventions féodales dans la Catalogne du $\mathrm{XI}^{\mathrm{e}}$ siècle », Structures sociales de l'Aquitaine, du Languedoc et de l'Espagne au premier âge féodal, Paris, Éditions du CNRS, 1969, p. 187 et suiv.
} 
que nous préférons pour l'instant nous arrêter; la raison en est que Godefroy propose, pour rendre compte des «diverses nuances de signification » du substantif droit dans son sens juridique un extrait d'un des plus importants coutumiers de Normandie :

L'en appelle aucunes fois droict la chose de quoy la possession appartient a aucun, si comme Paris est le droict au roy de France: aucunes fois appelle l'en droict satisfaction de tort faict a aucun, si comme l'en dit: Cestuy a eu droict de celuy qui le roba, quand il a esté pendu; aucunes fois appelle l'en droict le loyer que aucun a pour sa desserte, si comme l'en dict du larron qui est pendu, s'il a bien eu son droict; aucunes fois appelle l'en droict une vertu que rend a chacun ce qu'il doit avoir ; et aussy est appellee en cour laye droict, par quoy tous contends sont fines ; aucunes fois appelle l'en droict la voye de loyauté qui fine les querelles, si comme l'on dict: Celuy a fait droict qui loyaulment a jugé, ou finé une querelle. L'on appelle droict les loix et les coustumes de Normandie, pour ce que par eux est souvent le plet finé7.

Il s'agit là en réalité de la fin du premier chapitre du Grand coutumier de Normandie dont la première version, latine, du début du XIII ${ }^{\mathrm{e}}$ siècle, s'intitule Summa de legibus Normannie in curia laicali; ce court chapitre porte le titre De Jure et constitue effectivement un essai de définition de la notion de droit, définition dont Godefroy reproduit la partie la plus importante, dans une traduction plus tardive (le Grand coutumier de Normandie est une traduction postérieure à la mort de Saint Louis, de la fin du XIII ${ }^{\mathrm{e}}$ siècle donc). D'une façon tout à fait conforme à ce qu'affirmait déjà le prologue, l'auteur s'intéresse au droit comme mode possible de règlement des conflits et semble même le définir par son aptitude à mettre fin aux différends qui opposent les hommes («contents», « querelle», «plet») ${ }^{8}$; en effet, c'est parce qu'elles peuvent permettre de régler les désaccords, que les « lois et les coutumes » (de Normandie sans doute, mais peu importe) participent du droit; il y a là quelque chose de capital et nous ne croyons pas forcer le sens du texte en disant que c'est par analogie de résultat (règlement des contentieux) avec la «voie de loyauté » (equitatis linea) que ces «lois et coutumes»(leges et consuetudines) entrent dans ce qui est senti comme le droit; pour le dire autrement, le droit précède la loi ou ne prend la forme de la loi que finalement, ou en dernière instance?

C'est en gardant à l'esprit cette conception d'un droit dont la finalité sociale est de pacifier les relations humaines que nous étudierons les principaux recours à la notion de droit dans Girart de Roussillon; nous isolerons des séquences narratives

\footnotetext{
${ }^{7}$ Ancienne Coutume de Normandie, cit. par Godefroy, Dictionnaire de l'ancienne langue française, art. droict. Les italiques sont dans le texte.

${ }^{8}$ Dans le prologue l'auteur rappelle que c'est pour empêcher les effets destructeurs de la convoitise que Dieu a voulu que des princes règnent sur la terre, dictent des lois et mettent fin aux conflits que la discorde, qui est contraire à la paix, peut engendrer.

${ }^{9}$ Voici le texte latin original : Dicitur etiam jus, equitatis linea litem dirimens querelarum; secundum quod jus dicitur fecisse, qui aequitate servata litem terminavit. Dicuntur etiam jura, leges et consuetudines Normannie : eo quod querelae per eas frequentius terminantur.
} 
faisant apparaître chacune un problème de droit particulier (problème qui en soustend la composition ou qui en constitue la thématique principale).

La première séquence qui retiendra notre attention va de la reprise de la cité de Roussillon par Girart jusqu'à la bataille de Vaubeton : ce qui constitue l'enjeu de cette séquence, c'est la proposition de paix faite en vain par le héros à Charles. Il convient d'abord de rappeler - on verra bientôt pourquoi - l'accord initialement passé entre les deux protagonistes du conflit. Girart renonce à Elissent, qui lui était destinée, et reçoit Berthe en échange, que Charles devait épouser ; cet échange est assorti d'un accord dont la fonction est de compenser la perte et l'injure que représente pour Girart le fait de devoir renoncer à la cadette des deux sœurs, la plus belle et la plus désirable, que Charles exige d'épouser pour cette raison même, en dépit de ce qui avait été convenu avec leur père, l'empereur de Constantinople. Le mot utilisé pour désigner cet accord passé entre Charles et Girart est celui de «plait» (v. 485, 495, 533); les termes de l'accord en question sont réduits et parfaitement clairs : Girart est relevé de son hommage et tient désormais son fief en alleu; il s'agit d'un accord solennel, avec prestation de serment, et établi sous le contrôle de gens faisant autorité, de sages barons et des clercs ${ }^{10}$. L'abus de pouvoir de Charles, qui manifeste sa toute puissance à travers l'arbitraire de sa volonté, se solde donc pour lui par un accord politiquement paradoxal - il se prive de la dépendance d'un de ses plus puissants barons - que le personnage s'emploiera par la suite à nier ou à annuler.

L'accord est assorti, à la demande de Charles, de la reconnaissance d'un droit de chasse du souverain sur les terres de Roussillon - droit de chasse dont Charles ne tarde pas à profiter pour entrer sur les terres de Girart et pour s'emparer de la cité de Roussillon, grâce à la trahison d'un des hommes du comte. Girart convoque ses hommes, reconstitue une armée et reprend sa ville à la suite d'une bataille qui consacre la défaite de Charles, contraint de battre en retraite. Fouque, le neveu de Girart, recommande alors à son oncle, pourtant victorieux, d'entamer des pourparlers de paix avec le roi $^{11}$. Pendant le conseil consacré à cette question (v. 1458-1591), le mot « droit » apparaît quatre fois, dans un emploi identique :

Tramestes l'um message a son repaire [...], Quel reis vos mant son cuer e son viaire : S'el prend(e)ra vostre dreit senz loncestraire. E s'il aiquel soane, etc.

(v. 1483-1487)

Girart, s'el vol ton dreit, tu li feraz;

E s'il aichel soane, etc.

(v. 1527-1528)
Envoyez un messager à Charles, là où il se trouve [...], pour qu'il vous apprenne ses intentions et vous dise s'il accepte de s'entendre avec vous. S'il refuse, etc.

S'il veut bien te faire droit, Girart, tu accepteras. Et s'il refuse, etc.

\footnotetext{
${ }^{10}$ Voir v. 440-442 et v. 491-498.
}

${ }^{11}$ Aux yeux de Fouque, qui est une figure de la mesure et de la raison, cette paix s'impose à Girart en vertu de la souveraineté de Charles: "Carles est vostre sire, rius emperaire». (v. 1475). L'indépendance féodale n'exclut donc pas la sujétion de Girart à Charles. On ne voit pas le héros contester cette affirmation mais douter de l'utilité de négociations qui, selon lui, ne pourront déboucher sur rien vu l'état d'esprit de Charles. 
Sel reis non velt mon dreit e dis que non, etc. Si le roi refuse de me faire droit, etc. (v. 1536)

Presenterai ton dreit ab ist anel :

E n'i porterai letre, breu ne seel.

(v. 1566-1567)
Je lui présenterai ton offre d'entente avec cet anneau comme gage, - ni lettre, ni bref, ni sceau.

On le voit, pour rendre les emplois du mot «droit» précédé d'un possessif référant à Girart, Micheline de Combarieu et Gérard Gouiran font prévaloir tantôt l'idée d'une proposition de paix tantôt celle d'une reconnaissance du droit de Girart ${ }^{12}$. Mais il faudrait plutôt comprendre, selon nous, le mot «droit» de façon plus neutre, dans le sens impersonnel de «proposition conforme au droit»; il s'agit bien d'une proposition, puisque le «droit» en question doit être présenté au roi qui peut l'accepter ou le rejeter, mais cette proposition, faite par Girart (d'où le possessif) n'est pas directement ou immédiatement une demande de reconnaissance, par le personnage, de ses droits personnels : la démarche ne peut pas consister à exiger de Charles qu'il « fasse droit» au comte de Roussillon, ce qui serait une revendication et non une requête (dans cette histoire en effet, et d'une façon qui semble conforme à l'ordre politique des choses, c'est le comte de Roussillon qui est le requérant c'est à lui de demander la paix).

Le mot «droit» dans les occurrences citées plus haut prend tout son sens au moment où Fouque transmet la proposition de paix à Charles - message qui rapporte «le dreich/ de par Girart» (v. 1958-1959) ${ }^{13}$ et qui en définit du même coup explicitement, et en principe fidèlement, le contenu. Le personnage commence par assurer Charles que le comte de Roussillon est prêt à réparer ses torts dans le cas où il en aurait commis (v. 1960-1961), puis il entre dans le vif du sujet :

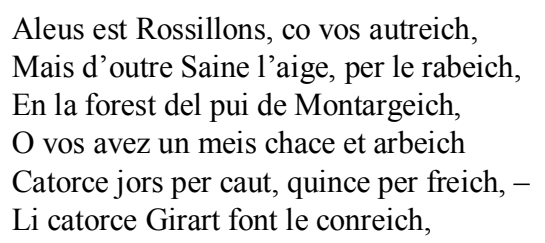

${ }^{12}$ Paul Meyer propose quant à lui une traduction littérale, laquelle a le défaut d'être peu éclairante quant à la nature du référent du mot « droit »; voir Girart de Roussillon, trad. cit., p. 45 : « il prendra votre droit »; p. 47 : « s'il veut ton droit »; id., : « si le roi ne veut prendre mon droit» et, p. $49:$ : je lui présenterai ton droit avec cet anneau ».

${ }^{13}$ L'expression apparaît déjà un peu avant, à l'occasion d'une anticipation faite par le narrateur:

Hui mais sera li mes au rei nunchaz,

De par Girart a Carle dreit presentaz. (v. 1917-18)

Selon les traducteurs, le message serait maintenant une proposition faite par Girart de « faire droit » à Charles. La situation n'a pourtant pas changé et le « droit» dont il est ici question est bien le même que celui de la scène de conseil dans le camp de Girart. 
Et l'aduiçent per Seine tote a naveich,

Lai o cist pin et laur estant ereich,

O meines ton deport et ton trepeich $[\ldots]^{14}$.

sur lequel il passe très rapidement (Roussillon est désormais un alleu) pour s'appesantir en revanche sur le droit de chasse et de gîte que Girart reconnaît volontiers à Charles, droit de gîte pris en charge par le comte de Roussillon, là même où Charles s'installe d'habitude pour se livrer à son divertissement. Mais il n'y a là - et le fait est d'importance - rien de plus que ce qui avait été déjà convenu entre les deux personnages à la suite de l'échange des épouses : le «droit» proposé par Girart, c'est donc tout simplement la proposition d'en revenir à l'accord initial, le contenu de ce «droit» correspondant exactement à celui du «plaid» dont nous parlions plus haut. Le droit que mentionne Fouque n'a pas de valeur générale, il n'est pas fait d'une loi ou d'un principe théorique à partir desquels il serait possible de juger du cas particulier représenté par le conflit entre Charles et Girart ; ce qui fait droit ou ce qui est institué en droit, c'est une convention particulière passée entre deux individus particuliers dans des circonstances particulières.

Il en va autrement du droit dont se targue Charles pour convoquer Girart après le meurtre de Thierry de Scanie par Boson d'Escarpion, le neveu du comte de Roussillon se vengeant ainsi de la mort de son père et de son oncle, l'un et l'autre tués par Thierry lors de la bataille de Vaubeton. Girart n'a aucune responsabilité dans un geste qu'il n'a pas commandité, ni même simplement autorisé, contrairement à ce que croit Charles qui veut tout de suite relancer la guerre contre son vieil ennemi. Il en est momentanément empêché par ses vassaux qui, à l'occasion d'un conseil tumultueux, parviennent à le convaincre, avant de déclencher des représailles militaires, de mander Girart en justice pour qu'il s'explique ; ce qui est alors demandé ou attendu du comte, c'est qu'il « fasse droit» à Charles (v. 3724, 3746, 3760, 3772). On envoie donc un messager, Pierre de MontAbei, pour transmettre l'injonction royale: Girart est mis en demeure de «faire droit » à son souverain, ce que ses plus sages conseillers l'invitent également à faire (v. 4044, 4051, 4096, 4509, 4515). Le comte de Roussillon ne veut rien entendre, refuse violemment de déférer à la convocation et fait même porter sur Charles toute la responsabilité de la situation. L'ambassade se solde par un échec, Girart n'ayant finalement pas voulu, comme Pierre de Mont-Abei le rapporte à Charles, venir lui «faire droit» (v. 4566, 4734). Toute la séquence est construite sur la possibilité d'une paix conditionnée par la réponse positive de Girart à une seule demande, « faire droit» à son souverain. La traduction littérale est certes commode, mais elle est ambiguë : elle suppose que le droit est du côté de Charles, ou que Girart est mis

${ }^{14}$ La Chanson de Girart de Roussillon, trad. cit., v. 1964-1972, p. 175 : « Certes, nous affirmons que Roussillon est un alleu, mais nous reconnaissons que vous avez un droit de chasse dans la forêt de Montargon, et droit de gîte pour un mois (deux semaines l'été, deux semaines l'hiver) pendant lequel Girart doit subvenir à vos besoins en vivres : il le fera en amenant par la Seine des convois de bateaux jusqu'au bois de pins et de lauriers où vous avez l'habitude de vous divertir. » (Les précisions géographiques du v. 1965 ne sont pas rendues) 
en demeure de reconnaître les droits de son ennemi, voire de réparer d'hypothétiques torts envers celui-ci.

La formule « faire droit» dans le sens d'apporter « satisfaction de tort faict a aucun » est attestée dans quelques occurrences ${ }^{15}$, quand Fouque s'adresse à Charles en faveur de Girart, prêt à participer lui-même à la réparation des torts de son seigneur :

S'el vos a fait leit tort que far non deich,

D'aico vos feran dreit aici meeich. (v. 1960-1961)

ou quand le comte de Roussillon propose un règlement fondé sur la reconnaissance mutuelle de torts qui seraient partagés :

E ferai li tot dreit, se l'ai fait mal,

E mon seignor reface mei autretal. (v. 4493-4494)

mais l'expression ne suppose probablement rien de tel dans les occurrences que nous signalions dans le paragraphe précédent. Ce qui permet de le dire, c'est une précision apportée à trois des occurrences en question :

Qu'el vos vegne $(m)$ dreit faire a vostre estage,

Si com ferent li ome de son linnage. (v. 3772-3773)

- Girart, Carles vos mande ista raison :

Que li annes dreit faire a ssa maison,

Eisi con tes lignages lo fes au son. (v. 4050-4052)

Mais manda ton seinor de ton viaire,

Que tu li f[e]ras dreit, con fest tos paire. (v. 4508-4009)

On demande donc à Girart de «faire droit» à Charles comme le faisaient avant lui les hommes de son lignage. On comprend que Girart est mis en demeure de déférer à la convocation de Charles en vertu de la souveraineté de ce dernier; dans la séquence construite autour de la demande faite à Girart de s'expliquer en justice sur le meurtre de Thierry de Scanie, «faire droit» équivaut à reconnaître le droit de Charles à exercer cette même justice, laquelle est justement de sa prérogative de souverain. C'est bien du droit du souverain, quel qu'il soit, à exercer la justice sur ses sujets dont il est question ${ }^{16}$ - déférer à la convocation de Charles, c'est

15 Pour l'expression «faire droit», le Tobler-Lommatzsch donne pour sa part « Recht sprechen » («parler de droit») mais précise, pour «faire droit a aucun d'aucune rien»: «jemandem für etwas Genugtuung geben » («donner réparation à quelqu'un pour quelque chose $»)$.

${ }^{16} \mathrm{C}$ 'est aussi à notre avis le sens qu'il faut donner à l'un des exemples proposés par le ToblerLommatzsch pour illustrer l'expression «faire droit a aucun d'aucune rien »- emploi strictement identique à celui qui nous intéresse ici : 
reconnaître son pouvoir de justice et c'est du même coup reconnaître sa souveraineté : si le refus de Girart suscite par la suite un accès de fureur chez Charles, c'est que ce refus est en lui-même un acte de rébellion.

Reste à s'interroger sur un autre emploi important du mot droit, dans deux expressions fort proches : «jugier droit» et « savoir de droit», qui peuvent d'ailleurs se combiner : «droit savoir jugier »; ces formules apparaissent à l'occasion de deux scènes de conseil où l'on voit Charles demander leur opinion aux plus avisés de ses vassaux :

Qui dreit nos sat jujar car le comance. (v. 1732)

Segnor, qui sat de dreit rien ni entent

Si m'en conselt per foi son escient. (v. 3566-3567)

et où l'on entend un conseiller faire valoir son avis par une sorte d'adage :

Om qui dreit sat jujar non dei mentir. (v. 3668)

Parlerait-on enfin d'une expertise juridique fondée sur une connaissance approfondie de règles et de principes susceptibles de trancher les questions débattues à l'occasion de ces conseils? Il n'en est rien: une fois de plus la notion de droit renvoie à autre chose qu'à une législation, fût-elle rudimentaire ou réduite à quelques lois identifiables.

La première scène de conseil dans le camp de Charles le montre exemplairement. L'objet en est d'examiner collectivement la proposition de paix faite par Girart, après que celui-ci a repris Roussillon. Charles ouvre la séance en demandant de s'exprimer à celui qui saurait bien parler du droit (v. 1732 cité plus haut: "dreit jujar», ou «jugier droit», i.e. "Recht sprechen» selon le ToblerLommatzsch). Thierry de Scanie prend immédiatement la parole pour reprocher à Charles de s'être emparé de la cité de Girart par trahison et non par les armes infamie que Dieu a punie en lui infligeant une défaite (v. 1734-1741); Isembart de Riom intervient alors pour défendre Charles, mais timidement : il est tout de suite contredit par Thierry qui fait porter la responsabilité des hostilités sur Charles et qui juge normale la réaction de Girart d'avoir voulu reprendre son bien (v. 1747-1757); l'argument de Thierry est repris par Enguerrand qui recommande ensuite d'accueillir favorablement la proposition de Girart, ce « conte franc naturau » qui leur envoie de surcroît un ambassadeur de grande valeur en la personne du noble et sage Fouque (v. 1761-1776). Le discours de ses barons met Charles dans une fureur extrême : il refuse de suivre leur avis et promet au contraire une guerre sans merci, affirmant sans fard sa volonté d'anéantir son ennemi, de s'emparer de ses terres et d'y apporter la mort et la désolation (v. 1785-1784). L'entêtement de Charles est mis par ses barons sur le compte de son orgueil; le personnage essuie alors à son tour la

Or volt saveir li reis se tu t'en volz venir

En sa curt devant li faire dreit e suffrir,

S. Thomas $W 5263$ 
colère de Thierry de Scanie qui lui reproche de ne pas écouter ses conseils, pourtant dictés par un scrupuleux respect du « droit» :

Mais per nul anemi qu'on mentoges

Ne dei estre de droit ne fel ne bles.

Car qui dreit fauserai faus traices es,

Et la cors o estait torne en defes.

Per tei ou di, Martel, qui dreit ne cres ;

Escoutes e esgardes, e rien ne ves

Plus que judeus Mesie qu'eu en croz mes ${ }^{17}$ !

Quel est donc ce droit dont parle Thierry de Scanie - droit que Charles ne veut pas «croire» (v. 1812) alors qu'il demande que son conseil en discute ? Les interventions des barons montrent que le «droit» discuté en conseil ne possède pas de contenu juridique lié à la féodalité. À la demande faite par Charles de parler de droit, les barons répondent en effet en parlant d'éthique et d'honneur, ce qui n'est pas répondre pour autant à côté de la question posée. C'est en réalité tout un système de valeurs que le roi n'a pas respecté et qu'il continue de bafouer - ce qu'on lui reproche; l'échange des deux sœurs inaugure du côté de Charles un manquement aux principes qui font la morale guerrière et/ou l'idéologie seigneuriale des protagonistes de la chanson : respect de la foi jurée et des accords conclus, recours à l'arbitrage des armes et non à de vils procédés comme la dissimulation et la corruption, ménagement de la susceptibilité naturelle des grands seigneurs (celle de Girart), prise en compte des gestes de bonne volonté (sa proposition de paix) et des manifestations d'honneur (l'envoi de Fouque, le plus valeureux de ses vassaux). Le droit auquel se réfère Thierry de Scanie n'est pas un ensemble de règles, de coutumes ou de lois établies et explicites mais renvoie à ce qui est perçu, par lui et par le groupe social auquel il appartient, comme juste et moral; le terme n'a pas de valeur absolue mais désigne l'ensemble des principes de probité et d'équité qui sont censés régir les relations sociales et politiques des membres les plus éminents de ce que nous appelons la société féodale.

Le Trésor de la langue française, dans la notice historique qui clôt l'article consacré au substantif «droit», signale deux occurrences du mot dans la Chanson de Roland, l'une dans le sens de «ce qui est permis ou exigible selon les principes d'une morale» et l'autre dans celui de «ce qui est permis ou exigible selon la législation ou des règlements officiels »; le terme est donc ambivalent et hésite entre la morale et la loi, comme d'ailleurs le bas latin directum dont il est l'avatar français, puisque le mot directum est d'abord attesté dans le sens général de «justice, application des principes du droit» $\left(\mathrm{VI}^{\mathrm{e}}\right.$ siècle) avant de l'être dans celui

${ }^{17}$ La Chanson de Girart de Roussillon, trad. cit., v. 1808-1614, p. 165 : « Mais ce n'est pas parce qu'il s'agit d'un ennemi que je dois transiger avec le droit ou le fausser. Car quiconque se comporte ainsi est un traître indigne et met sous le coup d'un interdit la cour où il réside. C'est pour toi que je le dis, Martel, pour toi qui rejettes ce qui est juste, pour toi qui as des oreilles pour ne pas entendre et des yeux pour ne pas voir, à l'instar du peuple juif qui ne voyait pas le Messie qu'il avait mis en croix !» 
de «règles du droit, ensemble des lois» (VIII ${ }^{\mathrm{e}}$ siècle). Le droit comme principe moral semble ainsi chronologiquement antérieur au droit comme législation; cette antériorité est encore attestée, mais cette fois-ci dans le champ des mentalités, par l'essai de définition que donne le Grand coutumier de Normandie qui, définissant le droit comme ce par quoi (en cour laïque) les contentieux sont réglés, évoque en effet la «voie de loyauté» avant «les lois et les coutumes», lesquelles - rappelons-le relèvent du droit parce qu'elles peuvent régler les différends au même titre que la voie de probité et d'équité évoquée juste avant.

L'appel au droit dans Girart de Roussillon est très majoritairement un appel à la probité et à l'équité (telles qu'elles sont du moins conçues par les protagonistes : il ne s'agit pas d'une morale universelle mais d'une éthique de classe), même quand la controverse entre les deux protagonistes du conflit ou leurs représentants soulève des questions de nature féodale (à l'exception peut-être de deux ou trois scènes, dans les camps de Charles ou de Girart, consacrées à la nécessité pour le comte de Roussillon de se soumettre à la justice royale ${ }^{18}$ ). La fréquence de la notion de «droit» et les controverses qu'elle suscite peuvent donner l'impression de longs débats juridiques mais l'emploi de l'adjectif « juridique» pour qualifier l'enjeu ou la nature de ces discussions n'est pas sans danger : si l'emploi du terme «droit» induit effectivement, dans notre texte, un ensemble de principes censés commander les comportements de façon contraignante pour les individus, à l'instar du droit au sens moderne du mot, la prégnance sur nos esprits de ce sens moderne peut donner l'illusion d'un monde régi, de même que le nôtre, par des règles et des lois dûment établies et clairement identifiables. L'analyse des principaux emplois du terme « droit» dans Girart de Roussillon montre que la notion de droit ne recouvre en rien une quelconque législation: elle ne réfère pas à un corps de lois ou de coutumes susceptibles de codifier les relations entre les protagonistes et n'établit pas à ce sujet de prescriptions ou d'interdictions formelles et catégoriques. Nous avons affaire à un monde où le droit semble se résumer, ou presque, à un simple système de valeurs -

\footnotetext{
18 Dans la première scène, les barons de Charles tentent de convaincre ce dernier de convoquer en justice Girart avant de lui faire la guerre : ils arguent de ce que l'on ne doit pas attaquer un vassal qui n'a pas nui lui-même directement à son seigneur et qui n'a pas clairement manifesté son refus de servir (v. 3668-3729). Dans la deuxième scène, en réponse à Pierre de Mont-Abei qui transmet le message de Charles, Girart prétend être victime de l'hostilité du souverain qui méconnaît ses droits et qui l'exclut ainsi de sa fidélité (v. 42894314), argument que le messager rapporte fidèlement à Charles quand il fait le compte rendu de son ambassade (v. 4662-4663) : l'hostilité du seigneur libère le vassal de son hommage ; c'est l'argument qu'avait par exemple utilisé Hugues de Lésignan, au début du $\mathrm{XI}^{\mathrm{e}}$ siècle, pour rompre son hommage au comte Guillaume V d'Aquitaine, lequel, pour pouvoir contester cette liberté revendiquée par son ancien vassal, avait consulté Fulbert de Chartres pour savoir en quoi consistaient au juste les obligations de celui qui a juré fidélité, la réponse de l'évêque de Chartres établissant durablement le contenu des devoirs et obligations des uns et des autres dans la société féodale. Voir O. Guillot, A. Rigaudière, Y. Sassier, Pouvoirs et institutions dans la France médiévale. Des origines à l'époque féodale, Paris, Armand Colin, 1993, p. 200-201 et J.-P. Poly, E. Bournazel, La mutation féodale, $X^{e}-X I I^{e}$ siècle, Paris, PUF, 1980, p. $147-150$.
} 
celui d'une haute aristocratie pour qui (ce qui complique les choses) le recours à la force constitue de surcroît une sorte de mode naturel d'action et de négociation.

Dans un tel contexte, rien ne fait obstacle à ce que Pierre Le Gentil qualifiait naguère de passions, à savoir l'orgueil et l'ambition, dans des formules qui laissaient entendre toutefois la possibilité d'un cadre juridique et institutionnel qui aurait pu les canaliser ou les étouffer ${ }^{19}$. La vérité est que ce cadre juridique et institutionnel n'a pas de contenu légal: ce n'est pas au droit, ni à la loi, que la volonté de puissance des deux protagonistes vient se heurter, mais à une éthique de classe de toute évidence insuffisante pour brider les exigences exorbitantes de leur idéal du moi. Les barrières morales, de probité et d'équité, sont insuffisantes pour faire contrepoids, et donc obstacle, à cette démesure morale que le texte appelle orgueil et qu'il attribue d'égale façon à Girart et à Charles.

Pour empêcher que ne s'installe la discorde entre les hommes, cette discorde qui est un effet de l'universelle convoitise, l'auteur de ce qui deviendra en français le Grand coutumier de Normandie rappelle, dans le prologue de son œuvre, que «Nostre Sire qui est Roy paisible et droicturier et ayme justice voulut que princes regnassent en terre, qui donnassent certaines loix de droict et finassent tous les contends, que discorde qui est contraire a paix peult engendrer. » Cet ordre-là, celui d'une concorde fondée sur une législation définie et garantie par la puissance royale, ne règne pas encore dans Girart de Roussillon, mais il commence peut-être à se construire, puisque la royauté l'emporte finalement sur celui qui en a contesté l'autorité et qui a ainsi exposé la collectivité au risque mortel de la guerre civile. L'avènement de cet ordre n'est cependant pas seulement conditionné par l'expulsion ou la reddition de celui qui l'empêche par sa démesure ; cet avènement dépend aussi d'une évolution du droit, non pas dans son contenu, mais dans sa nature, lequel doit en effet passer de l'éthique, toujours relative, à la loi, incontestable car absolue. Avant l'avènement de cet ordre-là, un baron révolté n'est jamais qu'un rebelle et un fauteur de troubles, mais il ne saurait être un hors-la-loi.

Philippe HAUGEARD

Université de Haute-Alsace, Mulhouse

ANR Juslittera

\footnotetext{
${ }^{19}$ P. Le Gentil, art. cit. p. 330 : «Partout et toujours on retrouve l'orgueil et l'ambition de Girard, partout et toujours le désir du roi d'abaisser un vassal devenu trop puissant, partout et toujours la faiblesse des institutions médiévales, impropres à maintenir ou à restaurer entre les deux hommes des attaches solides et durables »; ou encore, p. 466: «En réalité, les discussions juridiques sont maintenant dépassées et les passions commandent. »
} 